\title{
Effects of Treatment of Myofascial Trigger Points on the Pain of Fibromyalgia
}

\author{
Maria Adele Giamberardino - Giannapia Affaitati • \\ Alessandra Fabrizio • Raffaele Costantini
}

Published online: 5 May 2011

(C) Springer Science+Business Media, LLC 2011

\begin{abstract}
Myofascial pain syndromes (MPSs) from trigger points (TrPs) and fibromyalgia syndrome (FMS) are common musculoskeletal pain conditions that frequently coexist in the same patients. In recent decades, it has become evident that these entities greatly influence each other's clinical expression. FMS is mainly rooted in the central nervous system, while TrPs have a peripheral origin. However, the nociceptive impulses from TrPs may have significant impact on symptoms of FMS, probably by enhancing the level of central sensitization typical of this condition. Several attempts have been made to assess the effects of treatment of co-occurring TrPs in FMS. We report the outcomes of these studies showing that local extinction of TrPs in patients with fibromyalgia produces significant relief of FMS pain. Though further studies are needed, these findings suggest that assessment and treatment of concurrent TrPs in FMS should be systematically performed before any specific fibromyalgia therapy is undertaken.
\end{abstract}

Keywords Myofascial trigger points · Local injection . Fibromyalgia $\cdot$ Fibromyalgia syndrome $\cdot$ Pain syndrome . Peripheral pain generators $\cdot$ Myofascial pain $\cdot$ Pressure pain threshold · Musculoskeletal pain - Central sensitization . Comorbidity · Taut band - Anesthetic · Management . Pathophysiology $\cdot$ Tender point $\cdot$ Antidepressants $\cdot$ Local pain $\cdot$ Referred pain

\footnotetext{
M. A. Giamberardino $(\square) \cdot$ G. Affaitati $\cdot$ A. Fabrizio Pathophysiology of Pain Laboratory, Ce.S.I., "G. D'Annunzio" Foundation, University of Chieti,

Chieti 66100, Italy

e-mail: mag@unich.it

R. Costantini

Institute of Surgical Pathology, University of Chieti,

Chieti 66100, Italy
}

\section{Introduction}

Myofascial pain syndromes (MPSs) from trigger points (TrPs) and fibromyalgia syndrome (FMS) are common forms of musculoskeletal pain. Though distinct in diagnostic criteria and clinical features in typical cases (with regional pain in MPSs and generalized pain in FMS), these two entities very often can be confused with each other in clinical practice, mostly because of their frequent coexistence in the same patient. This can pose problems of differential diagnosis and treatment $[1 \bullet \bullet]$.

In recent decades, it has become increasingly evident that this coexistence is not a merely by-chance association, but rather reflects a cause-effect relationship. On one hand, in fact, TrPs are more frequent in FMS due to a higher susceptibility in FMS to microtraumatic events that are recognized causative factors for $\operatorname{TrP}$ formation [2]. On the other hand, muscle TrPs in any individual represent a powerful peripheral source of nociceptive impulses; in time, these are likely to favor the development of central sensitization phenomena that can promote the clinical manifestation of FMS in predisposed individuals [3].

These findings have raised the question as to whether treatment of TrPs in patients with the two conditions may have a significant impact on the pain of the more severe of the two: fibromyalgia. Though not yet very numerous, recent clinical studies have addressed this issue and found a significant benefit of local TrP extinction for the generalized FMS pain. This article provides updated information on this complex issue, summarizing the current knowledge about clinical features, pathophysiology, and classic treatment of MPSs/ TrPs and FMS separately; discussing the critical elements in differentiating the two in routine medical practice; and reporting the results of the studies showing the impact of MPS treatment on FMS symptomatology. 


\section{Myofascial Pain Syndromes from Trigger Points}

\section{Definition and Clinical Presentation}

MPSs are acute, recurrent, or chronic forms of regional musculoskeletal pain whose mean prevalences among middle-aged (30-60 years) men and women are $37 \%$ and $65 \%$, respectively, and $85 \%$ in elderly ( $>65$ years) adults $[4,5]$.

An MPS is a "complex of sensory, motor and autonomic symptoms caused by myofascial trigger points", where TrPs are "spots of exquisite tenderness and hyperirritability in muscles or their fascia, localised in taut, palpable bands, which mediate a local twitch response (LTR) of muscle fibres under snapping palpation and, if sufficiently hyperirritable, give rise to pain, tenderness and autonomic phenomena as well as dysfunction in areas usually remote from their site, called targets" [6]. Active TrPs provoke spontaneous pain, and thus, an MPS; latent TrPs are clinically silent but frequently evolve into active TrPs in time. A primary $\operatorname{TrP}$ is located in a muscle directly subjected to overload/repetitive overuse; a secondary $\operatorname{TrP}$ is induced in a muscle, neurogenically or mechanically, by the activity of a nociceptive focus in a different structure (deep somatic or visceral) $[1 \bullet \bullet$.

Diagnostic criteria for MPS derived from international multicentric studies or expert consensus meetings still are lacking. At present, the most clinically relevant features for diagnosing an MPS in current practice include identification of the taut band and reproduction of the spontaneous pain complaint by exquisite pressure on a point of tenderness within the band [7].

Essential steps in the approach to patients with suspected MPSs are clinical history collection and physical examination [8]. TrPs most often result from muscle traumas/ microtraumas; thus, patients should be questioned about any event, activity, or habit potentially causing muscle overload, overuse, or disuse. Possible secondary TrPs also should be explored by asking questions about any previous painful visceral disease or other deep somatic pain sources within the neuromeric field of the involved muscles $[9,10]$.

MPS pain, at rest and/or on movement, is tensive, constrictive, or cramplike and of variable intensity and duration, with sudden or gradual onset. It rarely is located in the TrP zone, but most often occurs in a distant area (target), typical of each muscle/TrP. Accompanying symptoms are altered muscle motor function, cooling, sweating, and pilomotor activation, or even lacrimation, imbalance, dizziness, and tinnitus for MPSs in the head/neck region [11].

At inspection during physical examination, the physician should look for any biomechanical discrepancy and asymmetry that may have caused muscle overload [9]. Palpation of muscles is first aimed at identifying the taut band through snapping or pincer techniques (muscle accessible from one or two directions, respectively) [8]. Both potentially elicit the "local twitch response" (LTR), a brief local contraction of muscle fibers. The $\operatorname{TrP}$ is identified as the point within the band of maximal tenderness upon firm digital pressure; this elicits pain locally and in the target if the $\operatorname{TrP}$ is sufficiently hyperirritable, and provokes a painful reaction by the patient, called "jump sign" $[4,8]$.

\section{Sensory Evaluation}

Hyperalgesia has been shown by decreased pain thresholds (pressure, electrical stimuli) at TrP level (in muscle, subcutis, and skin) and at target level (always in muscle, with extension to subcutis and/or skin only in high TrP hyperirritability) [12].

$\operatorname{TrP}$ treatment by injection (see below) produces desensitization not only locally but also in the target (thresholds significantly increased in skin, subcutis, and muscle), confirming the interdependence of the sensory changes at target level and the trigger activity [7, 13]. Areas outside the TrPs and targets show sensory normality in patients with MPS who have no concurrent pain conditions potentially affecting general pain sensitivity [14].

\section{Routine Laboratory and Instrumental Tests}

Specific tests for MPS identification still are needed, though some findings can be confirmatory of the clinical diagnosis (eg, spontaneous electromyography (EMG) activity at TrP, visualization of an LTR at ultrasounds) [6]. Microdialysis provides very interesting results (ie, high levels of pronociceptive substances in active TrPs), but the technique is not suitable for routine application in medical practice [15].

\section{Pathophysiology of Trigger Points}

A $\operatorname{TrP}$ would be a dysfunctional site where an abnormal increase is present in the production and release of acetylcholine packets from the motor nerve terminal under resting conditions (dysfunctional endplate). This mechanism would be enhanced by an initiating traumatic/microtraumatic event (primary TrPs) or referral process (secondary TrPs), with increased motor endplate activity, persistent release of acetylcholine, and sustained depolarization of the postjunctional membrane of the muscle fiber. This could, in turn, cause a continuous release and inadequate uptake of calcium ions from the local sarcoplasmic reticulum (SR), producing sustained shortening of sarcomeres. A vicious circle of hypoxia (with release of vasoactive/algogenic substances, responsible for local nociceptor sensitization, and thus, hyperalgesia), failed $\mathrm{Ca}^{++}$reuptake from the SR (due to energy impairment), and perpetuation of the contracture 
("integrated hypothesis" of the original "energy crisis" hypothesis) would thus be initiated [16].

\section{Management of Trigger Points}

Deactivation of TrPs must be combined with removal of perpetuating factors (eg, microtraumas, chronic infection, stress/mood disorders, poor sleep, and nutritional/metabolic imbalance) [6].

The most frequently applied techniques for $\operatorname{TrP}$ deactivation are spray and stretch, $\operatorname{TrP}$ pressure release, and local injection (see [7, 17] for a more detailed description of these and other techniques). The latter represents the gold standard; it can consist of "dry needling" or injection of active substances, particularly local anesthetics. The effectiveness of dry needling probably lies in the mechanical disruption of the integrity of dysfunctional endplates. However, injection of an analgesic markedly reduces the patient's discomfort during and after the procedure, and thus, enhances the global outcome of the therapy. Injection of any solution in general, including saline, may relieve symptoms temporarily by diluting and dissipating sensitizing substances in the region of the energy crisis [18]. Injection of botulinum toxin also is effective, but its efficacy is no greater than that of the local anesthetic; the latter, being much less expensive, is thus preferred [19].

\section{Fibromyalgia Syndrome}

\section{Definition and Clinical Presentation}

FMS is a chronic condition of widespread musculoskeletal pain and tenderness accompanied by numerous aspecific symptoms, among which sleep disturbance and affective dysfunction are particularly frequent. It affects about $4 \%$ of the general population; its sex distribution, nearly equal in childhood, is up to sevenfold more common in women than men in adulthood $(50-60 \mathrm{y})[1 \bullet \bullet]$.

Criteria for FMS diagnosis still remain those established by the American College of Rheumatology Committee in 1990 [20]: 1) a history of widespread pain (involving all limbs and the trunk) of at least 3-months duration; and, 2) tenderness to digital palpation (with a pressure of $4 \mathrm{~kg}$ ) in at least 11 of 18 (9 symmetrical) predetermined body sites called tender points (TePs). A TeP is a site of exquisite tenderness in soft tissues that, in contrast to the $\operatorname{TrP}$ of MPS, is not included in a taut band of muscle fibers, does not evoke LTR under snapping palpation, and, especially, does not refer pain at a distance when stimulated $[1 \bullet \bullet]$.

A critical revision of the above criteria is currently underway. The newly proposed criteria no longer consider the presence of TePs, but only assess clusters of clinical symptoms. Should they became unanimously recognized, we probably would witness a change in the profile of the typical fibromyalgia patient, with an expansion of the population receiving this diagnosis in the future [21].

The onset of FMS symptoms can be either gradual or post-traumatic (physical injury/psychological stress). The typical spontaneous pain is a persistent, diffuse, deep, and aching sensation in muscles, most often continuous with periodic exacerbations of high intensity (flares). Associated symptoms (eg, affective dysfunction, cognitive deficits, short-term memory loss, dizziness, syncope, nonrestorative sleep, daytime fatigue, prolonged morning stiffness, numbness, tingling, and dysesthesias) are present in various combinations. Comorbidity frequently occurs with depression, anxiety, irritable bowel syndrome, dysmenorrhea, interstitial cystitis, other rheumatic conditions, chronic fatigue syndrome, myofascial pain syndrome, low back pain, temporomandibular joint disorder, and headache. Patients with FMS report even light stimuli applied to their somatic structures as painful. As documented by many experimental studies, this is due to a generalized increased sensitivity to pain in terms of lowered pain thresholds of skin, subcutis, and muscle to a variety of stimuli (eg, mechanical, thermal, electrical, chemical) in both painful and nonpainful body areas [14, 22, 23].

\section{Pathophysiological Mechanisms}

Though the origin of the syndrome has not been completely clarified yet, there is a general consensus that the main disturbance behind FMS is an altered processing of the pain signal. On the whole, a number of neuroendocrine (ie, dysfunction of the hypothalamic-pituitary-adrenal axis), neurotransmitter (ie, altered metabolism of serotonin, norepinephrine, and dopamine, as well as substance $\mathrm{P}$, and nerve growth factor) and neurosensory (ie, central sensitization, abnormalities of descending inhibitory pain pathways) disturbances have been implicated in the generation of the syndrome. Patients with FMS have aberrant responses to pain on functional brain neuroimaging and also show an accelerated loss of brain grey matter, interpreted by some as a sign of premature aging of the brain [24]. A genetic predisposition would be essential for a variety of environmental stressors to lead to the clinical manifestations of FMS [22].

\section{Prognosis and Treatment}

Though not threatening the patients' life, FMS severely impacts on the quality of life and physical function [25]. Complete resolution of symptoms is rarely achieved, but a significant improvement is obtained with an adequate multimodal management program. 
After a comprehensive evaluation of pain, function, and psychosocial context of the patient, a multidisciplinary treatment approach should systematically be taken, including a combination of pharmacologic and nonpharmacologic interventions. Options for pain treatment include paracetamol or weak opioids, such as tramadol, while NSAIDs or corticosteroids are not recommended unless a coexisting inflammatory/autoimmune disorder is present.

Antidepressants are recommended for long-term treatment (cycles of several months) because they decrease pain and often improve function. Particularly employed are tricyclics (especially amitriptyline), but also selective serotonin reuptake inhibitors (eg, fluoxetine) or dual (serotonin and noradrenalin)-reuptake inhibitors (eg, venlafaxine, duloxetine, and milnacipran). Antiepileptics, especially pregabalin, also are recommended for pain treatment in FMS [26]. While the U. S. Food and Drug Administration has officially approved the use of three compounds, duloxetine, milnacipran, and pregabalin, for FMS, no official drug specific for the syndrome has been approved in Europe, where these compounds are currently used off-label for the syndrome, by the European Medicines Evaluation Agency [27].

Nonpharmacologic management includes heated-pool treatment, individually tailored exercise programs (eg, aerobic exercise, strength training), and cognitivebehavioral therapy. Relaxation, rehabilitation, physiotherapy, and psychological support also can be of help in many cases $[26,27]$.

One approach gaining progressively more importance in FMS management is treatment of the so-called "peripheral pain generators." It had been noted for quite some time in clinics that FMS patients with concurrent sources of nociceptive pain in their somatic periphery, such as an MPS from TrPs or a painful joint (a very frequent situation, given the high comorbidity level in FMS) [2], have exacerbation of their typical fibromyalgia pain [3]. This has suggested that, after appropriate identification, local suppression of these peripheral sources of nociceptive inputs may be beneficial not only for the local pain but also for the widespread symptoms of FMS $[1 \bullet \cdot$. An account of the recent studies specifically addressing this issue in the case of myofascial TrPs in FMS is provided below.

\section{Effects of Treatment of Myofascial Trigger Points in Fibromyalgia}

TrPs in muscles are recognized powerful sources of peripheral nociceptive impulses that can have profound influences on the sensory processing of painful messages at central level [28]. In this view, they can be potential activating/precipitating factors of conditions other than MPSs, particularly fibromyalgia.

As stated above, the coexistence of TrPs and FMS is very common epidemiologically, TrPs being significantly more frequent in patients with FMS than in the general population [29]. This probably happens because FMS patients, due to their chronic pain and disability and consequent poor posture/antalgic attitudes, are more prone to muscle microtraumas, well-known promoting factors for TrP formation. Once developed, TrPs can, in turn, enhance FMS symptoms through their input to the central nervous system [30]. Several authors have thus evaluated the possible contribution of TrPs to fibromyalgia symptoms, though with some methodological differences among studies.

Alonso-Blanco et al. [31••] systematically explored the presence of TrPs in multiple muscles in 44 patients with FMS versus 50 control subjects and determined whether the local and referred pain from active TrPs reproduced the overall spontaneous fibromyalgia pain pattern and whether widespread hypersensitivity (pressure pain thresholds [PPTs] at TePs sites) was related to the presence of widespread active TrPs. FMS patients had a mean of 11 TrPs (10 active, 1 latent) in contrast to control subjects only showing latent TrPs (mean: 2). The combination of the referred pain patterns from active TrPs fully reproduced the overall spontaneous pain area of FMS. Patients with FMS had significantly lower PPTs than control subjects and a significant positive correlation between the number of active TrPs and spontaneous pain intensity. The authors interpreted these results as an indication of the contribution of nociceptive inputs from active TrPs to central sensitization in FMS.

According to several authors, in addition to having more frequent TrPs than the normal population at all muscle levels, patients with FMS would commonly harbor TrPs at the very site of their TePs, with a significant overlap between TePs and TrPs [32, 33•]. In 2010, Ge et al. [33•] indeed tested the hypothesis that the 18 predetermined sites of TePs in fibromyalgia frequently are associated with myofascial TrPs and that the induced pain from active TrPs at TeP sites may mimic fibromyalgia pain. In their study of 30 patients with FMS, the TeP sites were specifically tested for trigger characteristics, both manually and at EMG recording of spontaneous activity, and the features of the spontaneous FMS pain were compared with those of the pain evoked by $\operatorname{TrP}$ stimulation. The authors found that most of the TeP sites were TrPs, with local and referred pain from active triggers partly reproducing the overall spontaneous pain pattern. The total number of active TrPs was positively correlated with the spontaneous FMS pain intensity. This study provided evidence in FMS patients of the importance of 
active $\operatorname{TrPs}$, which may serve as peripheral generators of fibromyalgia pain, and the authors suggested that inactivation of active $\operatorname{TrPs}$ may be an alternative for the treatment of FMS. Indeed, the year before (2009), a paper by Staud et al. [34•], in which the role of peripheral muscle input was explored in the initiation and maintenance of FMS, was published. In a randomized, doubleblind, placebo-controlled trial of 22 female control subjects and 28 female patients with FMS, the authors tested the effects of trapezius muscle TeP injections with $1 \%$ lidocaine on local pain thresholds and remote heat hyperalgesia in the forearm. Before muscle injections, shoulder pain was standardized by tonic mechanical muscle stimulation, resulting in local pain ratings of $4.0 \pm 0.5$ visual analogue scale (VAS) units. This stimulation was interrupted for the injections but continued afterwards at the same level. Both control subjects and patients showed significantly increased PPTs at the trapezius after lidocaine injections, but not placebo. Heat hyperalgesia in the remote site also was significantly reduced by lidocaine, but not placebo, in patients with FMS. Neither lidocaine nor saline injections significantly affected clinical FMS pain ratings, probably due to the very low dose of lidocaine employed $(50 \mathrm{mg})$. These results confirm the important role of peripheral inputs in maintaining central sensitization in fibromyalgia. In this study, the injection site was apparently not specifically tested for TrP characteristics. However, given the high frequency of coincidence of the trapezius TeP with a $\operatorname{TrP}$ area, it is highly probable that the beneficial effects of treatment in these patients were indeed due to the extinction of a TrP.

A recent paper by Affaitati et al. [35••] specifically evaluated the effects on fibromyalgia pain of treatment of concomitant TrPs that were not coincident with the TeP sites. They studied 68 female FMS patients with coexisting unilateral MPSs of the upper body, manifesting as accesses of regional pain from active TrPs in the trapezius (1 trigger in the medial third of the upper border, clearly distinct from the fibromyalgia TeP in the same muscle; $n=20$ ) or infraspinatus muscle ( 1 or 2 triggers; $n=48$ ).

In basal conditions, patients were assessed for their myofascial pain symptoms, (ie, number/intensity of pain episodes, PPTs at trigger site, paracetamol consumption), and FMS symptoms (ie, spontaneous diffuse pain [VAS] and hypersensitivity [PPTs at TePs sites, PPTs and electrical pain thresholds in skin, subcutis, and muscle in a distant, nonpainful area at quadriceps level]). They then were randomly assigned to two groups of 34 patients each to receive either active or placebo-like local $\mathrm{TrP}$ treatment (TrP injection with anaesthetic $[1 \mathrm{~mL}$ of $0.5 \%$ bupivacaine hydrochloride] or needle penetration in an area near the trigger) on days 1 and 4. Evaluations were repeated on days 4 and 8 .
The study protocol was double-blind, with neither patients nor clinicians collecting data and sensory evaluation being aware of the patients' group. Only the clinician performing therapy was unblinded. After treatment, in the active-treatment group but not placebo-treated group, number and intensity of myofascial episodes and paracetamol consumption decreased while PPTs at $\mathrm{TrP}$ site increased, with all changes being significant. In parallel, FMS symptoms also significantly improved; pain intensity decreased and all thresholds increased progressively in the TePs and nonpainful site. At day 8, all placebo-treated patients requested active local therapy, which was delivered on days 8 and 11, while none of the patients under active treatment requested additional therapy. At a 3-week followup (days 30 or 37), FMS pain still was lower than the initial, pre-study pain in patients not undergoing further therapy and had decreased in those receiving active therapy from day 8 .

The results of this study thus showed that in fibromyalgia patients with concurrent MPSs due to TrPs not coincident with TePs sites, local treatment of the peripheral muscle sources not only relieves regional symptoms but also produces a significant improvement of the widespread FMS symptoms (ie, lesser spontaneous pain and TePs hypersensitivity as well as reduced generalized hyperalgesia). The authors claimed that the diffuse tissue desensitization observed is not attributable to a systemic action of the drugs delivered locally to $\operatorname{TrPs}$ because $\operatorname{Tr} P$ anesthetic infiltration previously had been shown to not produce any significant change in pain thresholds in a nonpainful area in patients without fibromyalgia [13]. The decreased level of generalized hyperalgesia, as well as of spontaneous diffuse pain, must thus reflect other mechanisms related to the hypothesized pathophysiology of FMS (ie, a reduction of the degree of central sensitization). The extent of symptom decrease obtained in this study is not such to allow suspension of specific FMS treatments, but is sufficiently marked $(22 \%-30 \%)$ to hypothesize either a possible dose reduction of chronically administered drugs for FMS or a better symptom control at the same doses, with obvious advantages for the patients. The authors thus proposed that identification and treatment of peripheral pain generators represent the first approach to FMS before any other therapy is initiated. In this research, only FMS pain symptoms were tested; it will be important in future studies using a similar protocol to verify if other typical FMS complaints, such as sleep disturbance, fatigue, physical impairment, or affective dysfunction, also can actually benefit from extinction of peripheral nociceptive sources.

No other controlled study to date appears to have addressed the impact of treatment of clearly identified TrPs on FMS symptoms. However, a recent study by CastroSánchez et al. [36] has shown how local treatment of 
muscles, through massage able to release the TeP areas, has a positive impact on FMS symptoms. In view of the elevated number of TrPs in patients with fibromyalgia, it is probable that the observed beneficial effects of this treatment are secondary to TrP extinction. In this randomized controlled trial, 74 patients with FMS were randomly assigned to experimental (massage-myofascial release therapy) and placebo (sham treatment with disconnected magnotherapy device) groups for an intervention period of 20 weeks. Pain, anxiety, quality of sleep, depression, and quality of life were determined at baseline, after the last treatment session, and at 1 and 6 months. Immediately after treatment and at 1 month, anxiety levels, quality of sleep, pain, and quality of life were improved in the experimental group over the placebo group. Significant differences persisted at 6 months in the quality-of-sleep index. Myofascial-release techniques thus improved pain and quality of life in FMS.

On the whole, the results of the reported studies indicate that nociceptive muscle input in FMS, most often originating from TrPs, exacerbates FMS symptoms, and that reduction/extinction of this input substantially contributes to improving the fibromyalgia condition [37].

\section{Conclusions}

In synthesis, several recent studies have proven the important role of peripheral nociceptive muscle inputs in maintaining the diffuse level of sensitization in fibromyalgia, showing how local treatment of these sources is able, at least to some extent, to improve the diffuse symptoms of FMS. Not all of these studies involve a specific distinction between TrPs and TePs. Some research has provided evidence that local anesthetic infiltration of TePs is beneficial. However, because several well-controlled studies have shown that most TePs sites indeed coincide with areas of active TrPs, it is highly probable that the beneficial effects obtained on FMS are due to suppression of the input from TrPs. Other research in which a clear distinction was made between TePs and TrPs, with injections performed in TrPs not coinciding with TePs sites, also showed a significant improvement of the widespread pain and hypersensitivity of fibromyalgia. On the whole, the data so far available would indicate an important therapeutic effect of local treatment of TrPs in FMS, whether or not these sites coincide with those of TePs. If this local treatment provides a clear relief of FMS pain, its possible effects on other FMS symptoms, such as poor sleep, fatigue, or affective disturbances, still need to be investigated. In the examined studies, the improvement of FMS pain by $\operatorname{TrP}$ treatment is always partial, but of sufficient clinical relevance, both in extent and duration, to reason- ably forecast a dose reduction of specific drugs for FMS, and/or a better symptom control at the same doses. Thus, a systematic search for TrPs in FMS, at the TePs sites as well as in other locations, and their extinction is an approach that should systematically be adopted before any other therapy is initiated when faced with a patient with fibromyalgia.

Disclosures No potential conflicts of interest relevant to this article were reported.

\section{References}

Papers of particular interest, published recently, have been highlighted as:

- Of importance

•- Of major importance

1. .- Mense S, Gerwin RD (Eds): Muscle Pain. Diagnosis and Treatment. Heidelberg, Dordrecht, London, New York: Springer; 2010: 365 pp. This book provides a comprehensive description of musculoskeletal pain conditions, particularly myofascial pain syndromes from trigger points and fibromyalgia, and critically discusses their overlap, interaction, and implications for therapy.

2. Cummings M. Regional myofascial pain: diagnosis and management. Best Pract Res Clin Rheumatol. 2007;21:367-87.

3. Borg-Stein J. Management of peripheral pain generators in fibromyalgia. Rheum Dis Clin North Am. 2002;28:305-17.

4. Gerwin RD. Classification, epidemiology, and natural history of myofascial pain syndrome. Curr Pain Headache Rep. 2001;5:412-20.

5. Podichetty VK, Mazanec DJ, Biscup RS. Chronic non-malignant musculoskeletal pain in older adults: clinical issues and opioid intervention. Postgrad Med J. 2003;79:627-33.

6. Mense S, Simons DG, Russell IJ, editors. Muscle pain. Understanding its nature, diagnosis, and treatment. Philadelphia: Lippincott Williams \& Wilkins; 2001. p. 385.

7. Giamberardino MA, Affaitati G, Fabrizio A, Costantini R: Myofascial pain syndromes and their evaluation. Int $\mathrm{J}$ Clin Pharmacol Ther 2011; in press.

8. Simons DG, Travell JG, Simons LS (Eds): Travell \& Simons' Myofascial Pain and Dysfunction. The Trigger Point Manual, Vol 1. Upper Half of Body, $2^{\text {nd }}$ edn. Baltimore: Williams \& Wilkins; 1999:1038 pp.

9. Alvarez DJ, Rockwell PG. Trigger Points: diagnosis and management. Am Fam Physician. 2002;65:653-60.

10. Majlesi J, Unalan H. Effect of treatment on trigger points. Curr Pain Headache Rep. 2010;14:353-60.

11. Graff-Radford SB. Myofascial pain: diagnosis and management. Curr Pain Headache Rep. 2004;8:463-7.

12. Vecchiet L, Giamberardino MA, Saggini R. Myofascial pain syndromes: clinical and pathophysiological aspects. Clin J Pain. 1991;7:16-22.

13. Affaitati G, Fabrizio A, Savini A, et al. A randomized, controlled study comparing a lidocaine patch, a placebo patch, and anesthetic injection for treatment of trigger points in patients with myofascial pain syndrome: evaluation of pain and somatic pain thresholds. Clin Ther. 2009;31:705-20.

14. Vecchiet L, Giamberardino MA, de Bigontina P, Dragani L: Comparative sensory evaluation of parietal tissues in painful and 
nonpainful areas in fibromyalgia and myofascial pain syndrome. In Proceedings of the 7th World Congress on Pain, Progress in Pain Research and Management. Edited by Gebhart GF, Hansmond DL, Jensen TS. Vol 2. Seattle: IASP Press; 1994: 177-185.

15. Shah JP, Phillips TM, Danoff JV, Gerber LH. A in vivo microanalytical technique for measuring the local biochemical mileu of human skeletal muscle. J Appl Physiol. 2005;99:1977-84.

16. Kuan TS. Current studies on myofascial pain syndrome. Curr Pain Headache Rep. 2009;13:365-9.

17. Srbely JZ. New trends in the treatment and management of myofascial pain syndrome. Curr Pain Headache Rep. 2010;14:346-52.

18. Scott NA, Guo B, Barton PM, Gerwin RD. Trigger point injections for chronic non-malignant musculoskeletal pain: a systematic review. Pain Med. 2009;10:54-69.

19. Ho KY, Tan KH. Botulinum toxin A for myofascial trigger point injection: a qualitative systematic reviews. Eur J Pain. 2007;11:519-27.

20. Wolfe F, Smythe HA, Yunus MB, et al. The American College of Rheumatology 1990 criteria for the classification of fibromyalgia. Report of the multicenter criteria committee. Arthritis Rheum. 1990;33:160-72.

21. Wolfe F, Clauw DJ, Fitzcharles MA, et al.: Fibromyalgia Criteria and Severity Scales for Clinical and Epidemiological Studies: A Modification of the ACR Preliminary Diagnostic Criteria for Fibromyalgia. J Rheumatol. 2011 Feb 1. Epub ahead of print.

22. Abeles AM, Pillinger MH, Solitar BM, Abeles M. Narrative review: the pathophysiology of fibromyalgia. Ann Int Med. 2007;146:726-34.

23. Bennett RM. Clinical manifestations and diagnosis of fibromyalgia. Rheum Dis Clin North Am. 2009;35:215-32.

24. Robinson ME, Craggs JG, Price DD, et al.: Gray Matter Volumes of Pain-Related Brain Areas are Decreased in Fibromyalgia Syndrome. J Pain 2010 Dec 9. Epub ahead of print.

25. Giamberardino MA: Update on Fibromyalgia Syndrome. PCU 2008; XVI(4):1-6.

26. Carville SF, Arendt-Nielsen S, Bliddal H, et al. EULAR evidence based recommendations for the management of fibromyalgia syndrome. Ann Rheum Dis. 2008;67:536-41.

27. Sommer C: Fibromyalgia: a clinical update. PCU 2010; XVIII (4):1-4.

28. $\mathrm{Xu} \mathrm{YM}, \mathrm{Ge} \mathrm{HY}$, Arendt-Nielsen L: Sustained nociceptive mechanical stimulation of latent myofascial trigger points induces central sensitization in healthy subjects. J Pain doi10.1016/j. pain.2010.03.010.
29. Cakit BD, Taskin S, Nacir B, et al. Comorbidity of fibromyalgia and cervical myofascial pain. Clin Rheumatol. 2010;29:405-11.

30. Ge HY, Nie H, Madeleine P, et al. Contribution of the local and referred pain from active myofascial trigger points in fibromyalgia syndrome. Pain. 2009;147:233-40.

31. •• Alonso-Blanco C, Fernández-de-Las-Peñas C, Morales-Cabezas M, et al.: Multiple Active Myofascial Trigger Points Reproduce The Overall Spontaneous Pain Pattern in Women With Fibromyalgia and are Related to Widespread Mechanical Hypersensitivity. Clin J Pain. $2011 \mathrm{Feb} 28$. Epub ahead of print. This article demonstrates the high prevalence of active TrPs in multiple muscles of FMS patients, as compared to control patients, and the correlation between $\operatorname{Tr} P$ activity and FMS pain and hypersensitivity.

32. Ge HY. Prevalence of myofascial trigger points in fibromyalgia: the overlap of two common problems. Curr Pain Headache Rep. 2010;14:339-45.

33. • Ge HY, Wang Y, Danneskiold-Samsøe B, et al.: The predetermined sites of examination for tender points in fibromyalgia syndrome are frequently associated with myofascial trigger points. J Pain. 2010;11:644-651. This article shows that most of the TePs sites in FMS are TrPs. It underlines the role of TrPs as peripheral generators of fibromyalgia pain and suggests that their inactivation may be an important option for the treatment of FMS.

34. - Staud R, Nagel S, Robinson ME, Price DD: Enhanced central pain processing of fibromyalgia patients is maintained by muscle afferent input: a randomized, double-blind, placebo-controlled study. Pain 2009;145:96-104. This article emphasizes the important role of peripheral impulse input in maintaining central sensitization in FMS by showing a reduction of local and distant hyperalgesia upon anesthetic injection of the trapezius tender point.

35. •- Affaitati G, Costantini R, Fabrizio A, et al.: Effects of treatment of peripheral pain generators in fibromyalgia patients. Eur J Pain 2011; 15:61-9. This article provides clear evidence of the effects of local treatment of TrPs (not coinciding with the sites of TePs) on pain symptoms of fibromyalgia using a standardized experimental approach.

36. Castro-Sánchez AM, Matarán-Peñarrocha GA, Granero-Molina J, et al. Benefits of massage-myofascial release therapy on pain, anxiety, quality of sleep, depression, and quality of life in patients with fibromyalgia. Evid Based Complement Alternat Med. 2011;2011:561-73.

37. Staud R. Is it all central sensitization? Role of peripheral tissue nociception in chronic musculoskeletal pain. Curr Rheumatol Rep. 2010;12:448-54. 